\title{
Evolución de la colaboración médica cubana en 100 años del Ministerio de Salud Pública
}

\author{
Evolution of the Cuban medical co-operation during one \\ hundred years of existence of the Ministry of Public Health
}

\author{
Nestor Marimón Torres ${ }^{\mathrm{I}}$; Evelyn Martínez $\mathrm{Cruz}^{\mathrm{II}}$ \\ IMáster en Salud Pública. Especialista de II Grado en Administración de la Salud. \\ Ministerio de Salud Pública. La Habana, Cuba. \\ II Máster en Salud Pública y Salud Internacional. Especialista de I Grado en Medicina \\ General Integral. Ministerio de Salud Pública. La Habana, Cuba.
}

\begin{abstract}
RESUMEN
El 28 de enero de 2009 el Ministerio de Salud Pública de Cuba cumplió 100 años de su creación, el más antiguo del continente americano, y en ese marco se pretende describir la evolución y trascendencia de la colaboración médica cubana. Se fundamenta el desarrollo de la solidaridad desde la creación del Ministerio y como después de 1959 se convierte en un principio del Sistema Nacional de Salud. Se profundiza en las diferentes modalidades por la que ha transitado la colaboración médica y que se desarrollaron de acuerdo a las transformaciones económicosociales del país, la evolución cronológica de la historia de la colaboración médica y docente junto con los diferentes elementos que incidieron en sus modificaciones y perfeccionamiento. Se exponen las perspectivas de esta colaboración en el siglo XXI, centrada en la formación de recursos humanos de la salud como única vía posible para alcanzar un desarrollo sostenible de la salud de las poblaciones.
\end{abstract}

Palabras clave: Colaboración médica, solidaridad, evolución, recursos humanos en la salud, sostenibilidad, perspectivas. 
On January 28th 2009, the Ministry of Public Health of Cuba celebrated its 100th anniversary, being the oldest in the American continent. In this framework, it was intended to describe the evolution and importance of the Cuban medical cooperation and to substantiate the progress of solidarity since the inception of the Ministry and how this concept turned into a principle of the national healthcare system after the triumph of the Revolution. The different modalities of the medical co-operation, which developed according to the economic and social changes in the country, the chronological evolution of the history of the medical and teaching cooperation and the various elements that had an impact on the changes and the improvement of this co-operation were deeply analyzed. The prospects of this cooperation in the 21st century, centered on the formation of human resources in the field of healthcare, is the only possible way to attain sustainable development in the population's health.

Key words: Medical co-operation, solidarity, evolution, health human resources, sustainability, prospects.

\section{EL MINISTERIO DE SALUD MÁS ANTIGUO DEL MUNDO}

Durante la segunda ocupación norteamericana, (1906-1909) la Comisión Consultiva, organismo creado por el gobierno interventor en sustitución del Congreso de la República, al discutir y aprobar la Ley Orgánica del Poder Ejecutivo, incluyó entre las Secretarías de Despacho a la de Sanidad y Beneficencia. En esta decisión influyó el hecho de que el doctor Enrique Barnet Roque de Escobar, fuera el representante de la Jefatura de Sanidad y a su vez asesor de la Comisión Consultiva.

Esta ley fue promulgada por Decreto No. 78 del Gobernador Provincial y apareció publicada en la Gaceta Oficial el 26 de enero de 1909, para entrar en funciones dos días después al restaurarse en esa fecha la República burguesa.

De esta forma quedaba la organización de la salud pública cubana unificada, independiente y elevada a categoría ministerial para tener Cuba el privilegio de ser el primer país en hacerlo en todo el mundo, ya que otros lo lograron años después. Así, Austria elevó la suya en 1917; Checoslovaquia en 1918; Rusia después de la Revolución de Octubre; Gran Bretaña en 1919; Francia en 1920; República Dominicana y Líbano en 1921; Turquía en 1923, entre los primeros.

Los Estados Unidos de Norteamérica creó su ministerio después de 1940 y muchos países importantes como Alemania Federal, China, Dinamarca, Suecia, Noruega, Finlandia, España, Italia y Suiza tenían su organización de salud pública adscripta a otros ministerios.

Por la Ley Orgánica del Poder Ejecutivo, el Departamento Nacional de Sanidad y todas sus dependencias municipales, así como la Junta Superior de Sanidad, el Departamento de Beneficencia y la Junta Central de Beneficencia con sus juntas municipales e instituciones dependientes, pasaban a formar parte de la nueva Secretaría. En esta forma se mantiene la Secretaría hasta la puesta en vigor de la 
Constitución de 1940 en que se le dará una nueva estructura y cambiará su nombre por el de Ministerio de Salubridad y Asistencia Social. ${ }^{1}$

El año 1959, marcó un punto importante en esta evolución, momento en que triunfa la Revolución cubana y en el que se realizan importantes transformaciones políticas, económicas y sociales a favor del pueblo.

Dentro de estas transformaciones sociales se tomaron medidas concretas, dirigidas a mejorar la salud de la población en cumplimiento de los aspectos esenciales del Programa del Moncada, dentro de estas se encuentran: la reducción del precio de los medicamentos, la creación del servicio Médico Social Rural, campañas epidemiológicas, las transformaciones de la enseñanza superior y media de la medicina y la lucha ideológica en el Colegio Médico Nacional en los primeros años del triunfo. ${ }^{2}$

Como consecuencia de lo anterior, el Sistema Nacional de Salud sufrió una serie de transformaciones que permitieron alcanzar los logros que hoy ostentan y que para su mejor comprensión se han agupado en cuatro etapas:

I. De integración del Sistema Nacional de Salud único, que comprende 1959 y años de la década de 1960.

II. De consolidación del Sistema Nacional de Salud único, años de la década del 1970.

III. De incorporación y desarrollo de alta tecnología médica, impulso a las investigaciones y dispensarización de acciones de salud a toda la población del país a través del modelo de Atención Primaria del Médico y Enfermera de la Familia, años de la década de 1980.

IV. De lucha por la preservación de las conquistas logradas en el campo de la salud pública y por su desarrollo en condiciones de crisis económica, años de las décadas de 1990 y 2000.

El Sistema Nacional de Salud cubano, elevado a categoría ministerial hace 100 años y primero en el mundo, mantiene en la actualidad un ritmo permanente de desarrollo y perfeccionamiento, basado en sus principios, los cuales son:

- La salud es un derecho de la población.

- La salud de la población es responsabilidad del Estado.

- Los servicios de salud alcanzan toda la población por igual.

- Las prácticas de salud tendrán una sólida base científica.

- Las acciones de salud tendrán una orientación preventiva.

- La participación social es inherente al manejo y desarrollo de los servicios de salud.

- La solidaridad internacional será práctica de los servicios de salud. ${ }^{1}$ 


\section{APUNTES SOBRE LOS ORÍGENES DE LAS MANIFESTACIONES DE SOLIDARIDAD}

Los antecedentes de solidaridad internacionalista en la medicina cubana se remontan a la época de la colonia en la que algunos médicos de forma espontánea, expresan su espíritu revolucionario al partir a diferentes países para ofrecer su ayuda como profesionales o alistándose en sus ejércitos como soldados.

Se pueden enumerar algunos ejemplos como son: Dr. Antonio Lorenzo-Luaces de Iraola que participó con el grado de Coronel en la Guerra de Secesión Norteamericana, Dr. Manuel García-Lavín y Chapotín en la guerra franco-prusiana y en la que por los méritos alcanzados se le concedió la legión de honor de Francia y Dr. Luis Díaz Soto que participó en la lucha del pueblo español contra el fascismo en la guerra civil de 1936-1939, entre otros. ${ }^{3}$

Otro aspecto importante en este sentido lo constituye el apoyo y los aportes brindados por eminentes científicos cubanos en organismos internacionales.

En el año 1902 cuando la isla arriba formalmente a su independencia el 20 de mayo, pudo enviar una delegación a la I Convención Sanitaria Internacional de Washington celebrada del 2 al 4 de diciembre de ese año y en la que fue fundada la Oficina Sanitaria Internacional.

La dirección de la Oficina quedó integrada por un presidente, un secretario y cinco vocales; uno de los cuales lo fue el gran sanitarista cubano doctor Juan Guiteras Gener, jefe de esta delegación en la Convención y una de las principales figuras de la Oficina en sus próximos veinte años. De esta primera delegación también formaría parte el doctor Carlos J Finlay.

El doctor Guiteras representó a Cuba en las II y III Convenciones celebradas en Washington (1905) y México (1907), respectivamente. En 1909 se le cambió el nombre de Convención Sanitaria por el de Conferencia y así a la reunión llevada a cabo en San José de Costa Rica del 25 de diciembre de 1909 al 3 de enero de 1910, se le Ilamó IV Conferencia Sanitaria Internacional. ${ }^{4}$

Guiteras además de ser fundador de la Oficina, fue uno de sus dirigentes más importantes durante sus primeras dos décadas, fue uno de los que discutió y aprobó el Primer Código Sanitario Panamericano, ocupó una de sus

vicepresidencias, la presidencia de la Comisión de Fiebre Amarilla, vocal del comité de Tracoma, Beriberi, Meningitis Cerebro Espinal, siguió siendo reelegido como vocal de la oficina hasta que en el año 1921 fue nombrado Secretario de Sanidad y Beneficencia de la República de Cuba.

Otros médicos cubanos que desempeñaron una función importante en las primeras décadas de la Oficina Sanitaria Internacional fueron los doctores Hugo Roberts Hernández, Mario García-Lebredo Arango, Arístides Agramonte Simoni y Francisco María Fernández Hernández, entre otros que dieron su aporte en diferentes etapas. ${ }^{5}$

Estos son los ejemplos más relevantes del espíritu de solidaridad que desde épocas pasadas han destacado a los profesionales cubanos, pero no es hasta que se produce el triunfo revolucionario, en que estas demostraciones de ayuda solidaria y desinteresada se hacen más manifiestas, que se recogen antecedentes concretos e institucionales de ayuda en el campo de la salud con otros países.

\section{EL PRINCIPIO DE LA SOLIDARIDAD INTERNACIONAL}

http://scielo.sld.cu 
El pueblo cubano durante toda su historia ha tenido manifestaciones de solidaridad con otras naciones del mundo, demostradas desde los inicios del triunfo revolucionario, legados del pensamiento latinoamericanista de José Martí, Ernesto Che Guevara y Fidel Castro Ruz. La continuidad de estas ideas por este último durante todos estos años de revolución han sido la expresión del principio internacionalista del pueblo, expuesto de diferentes formas y vías, ejemplo son las asesorías técnicas en diferentes esferas, ya sean en la construcción, explotación y desarrollo de industrias, la agricultura, ganadería, avicultura, la pesca, el azúcar, la educación, el transporte, las comunicaciones y la salud. ${ }^{6}$

La revolución cubana no esperó su desarrollo económico y consolidación política para comenzar a brindar ayuda en el campo de la salud, aun con el éxodo masivo de médicos que se produjo a partir de 1959 cuando el país contaba con 6286 profesionales y de ellos emigró el $50 \%{ }^{7}$

A pesar de las acciones enemigas por tratar de desestabilizar la Revolución, en 1960 se envía una brigada médica emergente y varias toneladas de equipos e insumos a Chile, afectado por un intenso terremoto que dejo miles de fallecidos. Tal y como expresó Salvador Allende, entonces senador de ese país, de visita en Cuba y que acompaño a dicha brigada:

Vi los camiones pasando por las calles de La Habana y vi la generosidad anónima del que entrega lo que hace falta y que vale mucho más que lo que entregan los países ricos. $^{3}$

El 17 de Octubre del año 1962, Fidel anuncia al pueblo, en el acto de inauguración del Instituto de Ciencias Básicas y Pre clínicas "Victoria de Girón", la decisión del gobierno cubano de brindar ayuda en el campo de la salud, afirmando:

...y por eso nosotros, conversando hoy con los estudiantes, les planteábamos que hacen falta 50 médicos voluntarios para ir a Argelia, para ir a Argelia a ayudar a los argelinos.Y estamos seguros de que esos voluntarios no faltarán. iCincuenta nada más! Estamos seguros que se van a ofrecer más, como expresión del espíritu de solidaridad de nuestro pueblo con un pueblo amigo que está, peor que nosotros...Claro hoy podemos mandar 50, dentro de 8 o 10 años no se sabe cuántos, y a nuestros pueblos hermanos podremos darle ayuda; porque cada año que pase tendremos más médicos, y cada año que pase más estudiantes ingresarán en la Escuela de Medicina; porque la Revolución tiene derecho a recoger lo que siembra y tiene derecho a recoger los frutos que ha sembrado... ${ }^{8}$

Se considera entonces la fecha de inicio de la Colaboración Médica Internacional Cubana con brigadas permanentes el 23 de Mayo de 1963, con el envío de la primera brigada a Argelia, compuesta por 55 colaboradores que brindan sus servicios por un año. Aquí queda claramente expuesto el principio del internacionalismo en salud, de la solidaridad internacional del sistema cubano de salud pública y su fundamento ético, profundamente humanista, no como diplomacia médica, no como vehículo de difusión de una doctrina política y no como incentivo de relaciones comerciales o venta de servicios. ${ }^{9}$ (MINREX. Cooperación de Cuba en el mundo. Documento del Viceministerio de Cooperación Internacional, MINREX, La Habana, Cuba; 15 de Enero 2009).

Durante estos 49 años, el número de colaboradores y de países donde se brinda la ayuda se incrementa, se gana en experiencia y se mejora cada día más en calidad de la atención, se diversifica la cooperación médica en la mayoría de los campos de la salud y sobre todo en el área docente donde paulatinamente se abre un mayor número de facultades médicas en el exterior. De la misma manera, se ha 
comportado el número de estudiantes extranjeros en Cuba, de ahí que hasta la fecha se han graduado un total de 10241 estudiantes en las carreras de Medicina, Estomatología, Licenciatura en Enfermería y Técnicos de la Salud de 108 países hasta el curso escolar 2007-2008. (Informes de Archivos 1966-2009. Viceministerio de Docencia. Ministerio de Salud Pública). (Informes de Archivos 1993-2009. Unidad Central de Colaboración Médica).

En este período un total de 134849 colaboradores brindaron su ayuda en 108 países, agrupados por continentes de la siguiente forma:

- África: 38 países con 45488 colaboradores.

- América: 39 países con 79717 colaboradores.

- Euro-Asia y Medio Oriente: 31 países con 9644 colaboradores.

Hoy existe colaboración médica en 74 países y 38538 trabajadores de la salud cumplen con esta responsabilidad. ${ }^{10}$ (Informes de Archivos 1966-2009. Viceministerio de Docencia. Ministerio de Salud Pública). (Informes de Archivos 1993-2009. Unidad Central de Colaboración Médica).

En todos estos países, la colaboración médica se mantuvo y mantiene en forma totalmente desinteresada y se rige por los siguientes fundamentos básicos:

- La colaboración constituye un eje importante de la política exterior cubana como vía para la verdadera integración.

- Se canaliza a través de gobiernos centrales y locales y se busca crear o fortalecer una infraestructura que permita su sustentabilidad.

- Se desarrolla a partir de la suma de potencialidades entre los países involucrados, sin fines de lucro, teniendo en cuenta las diferencias y asimetrías existentes.

- Se envían profesionales y técnicos de la salud que se dedican fundamentalmente a la atención primaria, prestan sus servicios a toda la población sin distinción de razas, credos e ideologías, sin mezclarse en asuntos de política interna y respetando las leyes y costumbres de los países donde laboran.

- Sigue la máxima de responder a las necesidades planteadas por los países receptores de la ayuda.

- Por su naturaleza institucional intenta beneficiar el mayor número de población posible y se desarrolla en las zonas más remotas y postergadas. ${ }^{11}$

Cuba representa con ello un precedente sin paralelo en la historia de las relaciones internacionales, si se toma en cuenta que el país no ha alcanzado aún un completo desarrollo económico. ${ }^{9}$

Este incremento sostenido de la colaboración medica en general y la docente en lo particular fue posible también por el desarrollo y fortalecimiento que adquiriera el Sistema Nacional de Salud. Basta señalar que de 3 mil médicos en los primeros años de la revolución, hoy existen más de 72 mil, de ellos 32548 son especialistas de Medicina General Integral y de una sola Facultad de Medicina que pertenecía a la Universidad de la Habana, hoy existen 22 Facultades de Medicina incluyendo la 
Escuela Latinoamericana de Medicina. (Informes de Archivos 1993-2009. Unidad Central de Colaboración Médica).

El escenario docente se ha extendido, no solo a las provincias que tienen sus escuelas, sino también a los municipios y policlínicos que ya tienen sus sedes universitarias, pues las transformaciones que se producen en la enseñanza han permitido que el país completo sea hoy una gran universidad.

\section{DESARROLLO Y EVOLUCIÓN DE LA COLABORACIÓN MÉDICA CUBANA}

El desarrollo y evolución de la colaboración médica pasa por diferentes etapas que están condicionadas por factores externos e internos o ambos, que repercuten en el contexto nacional.

Su evolución por décadas es de la siguiente forma: etapa de los 60, se inicia la colaboración en la modalidad de misión internacionalista, que se basa en el principio de ayuda solidaria gratuita y estuvo potenciada por los diferentes movimientos de liberación que se desarrollaron en África y Centroamérica. De esta misma forma se comportó en los 70 y 80 . Ejemplos de países con colaboración en estos años fueron Argelia, Angola, Etiopía y Nicaragua,

La década de los años 90, estuvo marcada por eventos externos que afectaron sobremanera la economía cubana, dentro de ellos, la desintegración de la URRS con la desaparición del campo socialista, principal mercado cubano, el inicio del período especial y el recrudecimiento del bloqueo económico de Estados Unidos hacia Cuba.

Esta situación potencia el surgimiento de una nueva modalidad de cooperación: la Asistencia Técnica Compensada o Contrato Directo, cuya esencia consiste en que se establece un acuerdo, por el cual el médico contratado percibía una remuneración por los servicios prestados y por este concepto entraba al Sistema Nacional de Salud un aporte que permitía mantener al resto de la colaboración médica, que debido a la situación económica que existía, el país no lo podía asumir.

Al final de los 90 se producen eventos naturales en el área de Centroamérica y el Caribe (huracanes George y Mitch) que modificaron todo lo que se realizaba hasta estos momentos, disminuye la modalidad de Misión Internacionalista, la Asistencia Técnica Compensada se reduce de forma paulatina y aparece el Programa Integral de Salud (PIS), el 3 de noviembre de 1998, inicialmente en Centroamérica y EI Caribe y posteriormente se extiende hacia África y el Pacífico, así como la creación en este periodo de la Escuela Latinoamericana de Medicina, como elemento básico de continuidad y sostenibilidad de este Programa.

La esencia del PIS es el envió de Brigadas Médicas hacia lugares remotos, de difícil acceso, donde no había presencia de médicos nacionales y a los que solo se les proporciona un dinero de bolsillo para las necesidades básicas.

El nuevo milenio, con el impulso y desarrollo de la Revolución Bolivariana en Venezuela, se convierte en un factor externo que propicia la aparición de la modalidad de Programas Especiales, este fue Barrio Adentro el 16 de Abril del año 2003, modalidad más avanzada y completa (en términos de servicios brindados dentro de un país), actualmente como expresión de la Alternativa Bolivariana para las Américas (ALBA). ${ }^{11}$

Otro importante suceso es la creación del contingente internacional de médicos especializados en situaciones de desastres y graves epidemias "Henry Reeve", el 25 de agosto de 2005 por iniciativa de Fidel Castro Ruz. Este contingente se organiza 
como consecuencia de otro desastre natural; el huracán Katrina, que afectó a los territorios de Lousiana, Mississippi y Alabama en Estados Unidos y constituye un nuevo enfoque de la Medicina de Desastres, aunque a partir de 1960 ya Cuba brindaba esta ayuda ante desastres naturales a través de Brigadas Emergentes. ${ }^{12,13}$

Hasta la fecha, 10 mil cooperantes cubanos integran el contingente "Henry Reeve", y 4156 de ellos ya cumplieron misiones en siete países: Guatemala, Pakistán, Bolivia, Indonesia, México, Perú y China, con importantes resultados en su trabajo. ${ }^{10}$ (Informes de Archivos 1993-2009. Unidad Central de Colaboración Médica).

La evolución histórica de la colaboración médica por décadas se resume en la figura. ${ }^{11}$

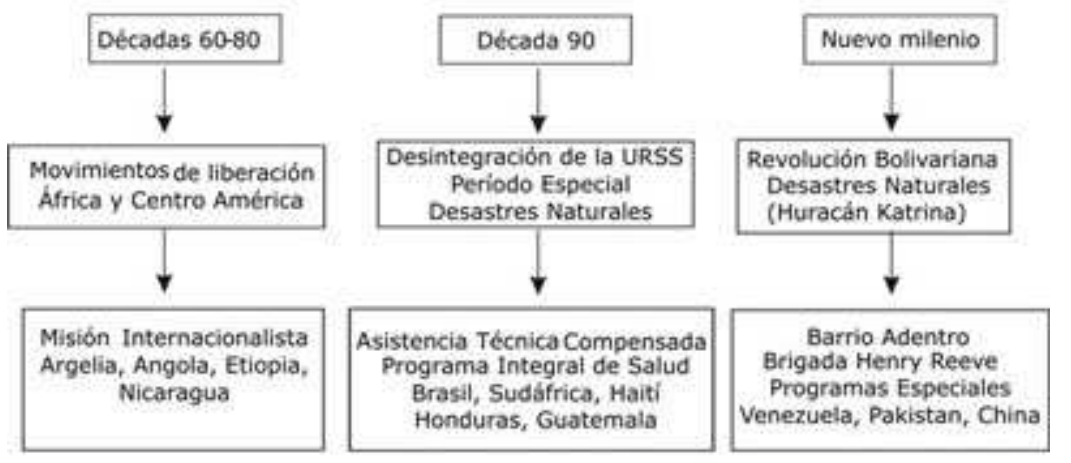

Fig. Evolución histórica de la Colaboración por décadas.

Otro programa importante que amplía las posibilidades y perspectivas de la ayuda cubana en el campo de la salud en el exterior es la "Operación Milagro", que se inicia en el año 2004 cuando el 8 de julio llegan los primeros pacientes de Venezuela, el 22 de julio del 2005 se ofrece a 15 países del Caribe y el 15 de septiembre del mismo año se extiende a 12 países de América Latina.

Desde su inicio hasta enero del 2009 se realizaron 1425921 intervenciones quirúrgicas a pacientes de 33 países, de estos 172613 han sido operados en Cuba y 1253308 en 51 centros oftalmológicos instalados por Cuba en 12 países. ${ }^{10,13}$ (MINREX. Cooperación de Cuba en el mundo. Documento del Viceministerio de Cooperación Internacional, MINREX, La Habana, Cuba; 15 de Enero 2009).

La voluntad por garantizar la continuidad y el perfeccionamiento de las acciones realizadas hasta el momento, son los pilares básicos del éxito de esta hermosa tarea. Los países que reciben la ayuda reflejan mejores indicadores de salud que se pueden considerar como resultados de estos esfuerzos.

Por ejemplo, solo en los primeros diez años de colaboración en el PIS en 42 países, se han realizado más de 457 millones de consultas médicas, de estas atendidos en terreno 174 millones 732 mil 736 pacientes, 954919 partos realizados, 2 millones 832 mil 973 intervenciones quirúrgicas y más de 2 millones de vidas salvadas. ${ }^{10,14}$ (Informes de Archivos 1993-2009. Unidad Central de Colaboración Médica).

La reducción de las tasas de mortalidad infantil y materna son indicadores que demuestran la relevancia del trabajo que se realiza. Ejemplos de países como Guatemala que a la llegada de la brigada presentaba una tasa de mortalidad infantil de $45 \times 1000$ nacidos vivos (NV) y durante este período se redujo a $16 \times 1000 \mathrm{NV}$ en el área atendida por la colaboración médica, en Gambia de $121 \times 1000$ NV a 
40,6 $\times 1000 \mathrm{NV}$; también la reducción de las tasas de mortalidad materna, ejemplos Haití de 457 x 10000 NV a 285 × 10000 NV, Ghana de 214 × 10000 NV a $43 \times 10000$ NV. ${ }^{10}$ (Informes de Archivos. 1998-2009. Departamento Nacional de Estadísticas. MINSAP).

El Programa Especial de Cooperación con Venezuela, en 5 años de desarrollo a través del trabajo realizado por sus diferentes modalidades: Barrio Adentro I, Estomatología, Ópticas y Barrio Adentro II compuesto por 483 Centros de Diagnóstico Integral, 26 Centros de Alta Tecnología y 548 Salas de Rehabilitación Integral, presentan como principales resultados los siguientes: 332 millones 616 mil 379 consultas médicas, 74 mil 398 intervenciones quirúrgicas, 6 mil 800 partos, 475 mil 393 vidas salvadas y 19 millones 126 mil 451 pacientes en rehabilitación. ${ }^{11}$ (Informes de Archivos 1993-2009. Unidad Central de Colaboración Médica).

Además, como parte de las acciones de Cooperación se realizó el Estudio Nacional de Discapacidad, cuyo principal impacto es el social ya que permitió diagnosticar y tomar decisiones encaminadas a mejorar la salud de las personas necesitadas.

Toda la panorámica y el resumen histórico antes señalado demuestran la necesaria evolución, perfeccionamiento y desarrollo de los objetivos o líneas de trabajo de la colaboración médica. En los inicios, la ayuda se basaba esencialmente en la asistencia médica. Posteriormente se realizaron diferentes actividades encaminadas a la capacitación y formación de recursos humanos y actualmente la asistencia médica per se no es su elemento fundamental, pues la formación de recursos humanos gana espacios cada vez más importantes dentro de ella, combinándose o integrándose a la labor asistencial mediante el desarrollo de Facultades de Medicina, la capacitación a líderes de la comunidad (formales e informales), la formación de técnicos, las asesorías en diferentes Ministerios de Salud y como aspecto crucial, la aplicación del Nuevo Programa de Formación en Medicina con buenos resultados a través de las Brigadas Médicas.

No se puede perder de vista la consideración de la salud como un recurso imprescindible para el desarrollo en cualquier contexto. Al mismo tiempo la formación y el perfeccionamiento de los recursos humanos en los sectores sociales y sanitarios constituyen un componente preciso para el desarrollo sostenible de la sociedad. Y en este caso la formación y capacitación de los recursos humanos para la salud son factores esenciales para garantizar la propia continuidad de la colaboración médica. ${ }^{14}$

Un resultado concreto y relevante es la participación de 534305 personas en las diferentes actividades de capacitación y formación de recursos humanos, de ellos, 220729 participaron en cursos y adiestramientos, 895 son auxiliares técnicos graduados, 1054 técnicos formados y 304 profesionales de la salud y de estos 278 son médicos. ${ }^{10}$ (Informes de Archivos 1993-2009. Unidad Central de Colaboración Médica), (Informes de Archivos. 1998-2009. Departamento Nacional de Estadísticas. MINSAP).

Durante los 49 años de historia de la colaboración, la formación de recursos humanos es un elemento primordial y como se explicó anteriormente, indisoluble de la asistencia médica; la apertura de 11 escuelas de medicina en el exterior con personal cubano así lo demuestra. En orden cronológico, la primera fue en Yemen del Sur en 1976, Guyana 1984, Jimma (Etiopia) 1984, Guinea Bissau 1986, Mbarara (Uganda) 1988, Thamale (Ghana) 1991, Huambo (Luanda) 1992, Gambia 2000, Guinea Ecuatorial 2000, Haití 2001 y Eritrea 2003. ${ }^{11}$ (Informes de Archivos 1966-2009. Viceministerio de Docencia. Ministerio de Salud Pública). (Informes de Archivos 1993-2009. Unidad Central de Colaboración Médica). 
La Escuela Latinoamericana de Medicina (ELAM), inaugurada en Noviembre de 1999, fue la alternativa diseñada para lograr la continuidad del PIS, como su complemento y sostenibilidad. A esta institución ingresan estudiantes de las regiones más postergadas, de familias humildes y de diferentes grupos étnicos, dotados al egresar de una buena formación desde el punto de vista científico, capaces, competentes, con una inclinación hacia la atención primaria de salud y grandes valores humanos.

Se han efectuado cuatro graduaciones de esta escuela, la primera fue en septiembre del 2005, con un total de 1611 médicos, la segunda con 1 592, la tercera con 1841 y la cuarta de 1669 profesionales. En el curso escolar 2008-2009 existe una matrícula de 1933 estudiantes pertenecientes a 18 países de América Latina y El Caribe. ${ }^{15-18}$ (Informes de Archivos 1966-2009. Viceministerio de Docencia. Ministerio de Salud Pública). (Informes de Archivos 1993-2009. Unidad Central de Colaboración Médica).

En el año 2004 como parte de las profundas y esenciales transformaciones que se producen dentro del Sistema Nacional de Salud para continuar elevando la calidad de los servicios que se brindan a la población, se comienza un nuevo modelo formativo de la carrera de medicina, que utiliza como medios de enseñanza las tecnologías de la información y las comunicaciones, tiene como base al Policlínico; estructura fundamental de la Atención Primaria de Salud, con el enfoque de formación desde los servicios para mejorar su calidad y con el principio de la educación en el trabajo como esencia en esta forma de enseñanza. Al mismo se le denomina Nuevo Programa de Formación en Medicina, comenzó en 75 policlínicos del país los que se convirtieron en Policlínicos Universitarios y hoy se aplica este método en todo el territorio nacional (Informes de Archivos 1966-2009. Viceministerio de Docencia. Ministerio de Salud Pública).

Esta experiencia, por las ventajas que posee, se comienza a aplicar para estudiantes extranjeros dentro y fuera de Cuba con la realización de las adecuaciones necesarias, con profesores cubanos y ya existen avances importantes.

El 21 de Agosto del 2005, se celebra en el extremo más occidental del país, la inauguración de Villa "Bolívar"construida con la cooperación de Venezuela y en presencia de los presidentes Fidel Castro Ruz y Hugo Chávez Frías y en este marco se establece el "Compromiso Sandino", con el propósito de formar en Cuba 200000 médicos en un periodo de 10 años para Latinoamérica, esta experiencia comenzó en el 2006 y hoy existen 14542 estudiantes distribuidos en cinco provincias del país. ${ }^{10,18}$ (Informes de Archivos 1966-2009. Viceministerio de Docencia. Ministerio de Salud Pública).

Otras experiencias relevantes en la aplicación de este Programa son:

- Formación de estudiantes de Medicina Integral Comunitaria en Venezuela, que cuenta con una matrícula de 20578 alumnos distribuidos en primero, segundo y tercer año de la carrera.

- Nuevo Programa de Formación de médicos en países del PIS. Esta modalidad forma 365 jóvenes vinculados a la atención médica de los habitantes de sus propias comunidades, en los siguientes países: Guinea Bissau con 138 estudiantes, Timor Leste con 147, Gambia con 40 y Tanzania/Zanzíbar con 40.

Cuba apoya, además de la formación de médicos, la formación de enfermeras en El Caribe por la crítica situación con este recurso en La Región, ya que existe una gran 
migración de este segmento profesional hacia países desarrollados y para tratar de suplir esta crisis se acuerda la creación en el año 2005 del Programa de Formación de Enfermeras para el Caribe, con la apertura de Escuela de Enfermería de Dominica, reinaugurada por el Primer Ministro Roosvelt Skerrit en febrero del 2006, tiene una matrícula actual de 127 estudiantes y cuenta en su claustro con 17 profesionales. Por otro lado, en Cuba se forman en estos momentos, en varios centros de salud 353 jóvenes en esta carrera procedentes de países de CARICOM. ${ }^{10}$

Hasta aquí se ha visto la evolución histórica de la colaboración médica a lo largo de los 100 años de constitución del Ministerio de Salud Pública de Cuba, con su máxima expresión en los últimos 50 años de su desarrollo, período también en que se han producido las grandes transformaciones sociales, políticas y económicas del país con el triunfo de la Revolución.

Los propósitos y proyecciones de la colaboración médica no solo han estado condicionados por los intereses propios de Cuba sino por una visión que va más allá de las fronteras del país y que tiene como principio la ayuda desinteresada y la intención de apoyar donde realmente hace falta.

\section{EL PENSAMIENTO DE FIDEL}

Cuando se habla de esta importante obra: la Colaboración Médica, que comenzó hace 49 años y de la cual todo el pueblo cubano se enorgullece no se puede dejar de mencionar a Fidel Castro Ruz como su principal ideólogo e impulsor, no existe un momento en su desarrollo que no haya estado marcado por su pensamiento y al que no le haya dado un seguimiento personalizado hasta el más mínimo detalle, se puede afirmar sin ningún tipo de dudas que Fidel es el autor de toda esta importante labor y de la que siempre está pendiente con el ánimo de fortalecerla y perfeccionarla cada día más.

En su reflexión del 3 de marzo de 2008, resume de forma muy específica los principios de esta ayuda solidaria:

Los médicos y los demás profesionales y técnicos de la salud cubanos constituyen una fuerza excepcional. Ningún país cuenta con algo similar; igual que los soldados internacionalistas de nuestra isla, se formaron en el combate. Sus misiones en el exterior se atienen a rigurosas normas éticas. Sus servicios se prestan gratuitamente o se comercializan según las circunstancias del país receptor. Ellos no son exportables. ${ }^{19}$

En otra más recientemente afirma: "Los profesionales de la salud han escrito con letras de oro páginas imborrables de la historia." 20

Muchos son los momentos en que en sus palabras expresa las bases que sustentan esta ayuda solidaria y un ejemplo es el siguiente:

Alrededor de 35 mil especialistas cubanos de la salud prestan servicios gratuitos o compensados en el mundo. Adicionalmente, un número de jóvenes médicos de países como Haití y otros de los países más pobres del Tercer Mundo laboran en su patria por cuenta de Cuba. En el área latinoamericana fundamentalmente contribuimos con operaciones oftalmológicas que preservarán la vista a millones de personas. Estamos, por otra parte, contribuyendo a la formación de decenas de 
miles de jóvenes estudiantes de medicina de otras naciones, en Cuba o fuera de Cuba.

No es algo, sin embargo, que arruine a nuestro pueblo, que pudo sobrevivir gracias al internacionalismo que la URSS aplicó con Cuba, y nos ayuda a pagar nuestra deuda con la humanidad. ${ }^{21}$

El Ministro de Salud José Ramón Balaguer Cabrera, en la celebración del 45 Aniversario de la Colaboración Médica y el $10 \mathrm{mo}$. del PIS, destaca que la base del Sistema Nacional de Salud cubano es la atención primaria, cuyas raíces se ubican en las ideas de "La Historia me absolverá" y que tuvo en los médicos guerrilleros de la Sierra Maestra a sus primeros protagonistas y ratifica que el inspirador principal de todas estas acciones es Fidel. Lo anterior queda sintetizado en el reconocimiento que se le realizó, consistente en un diploma con una fotografía suya en la que aparece donando sangre para socorrer a víctimas del terremoto en Perú, en julio de 1970 , gesto que fue seguido por otros más de 100000 cubanos. ${ }^{22}$

De esta forma sencilla y emotiva se reconoce públicamente una vez más, el decisivo papel de guía e impulsor de todas las hermosas obras que se realizan en Cuba desde el año 1959, de las cuales la Colaboración Médica es una de las que mayor repercusión tiene fuera de las fronteras cubana.

\section{LA COLABORACIÓN MÉDICA DE CARA AL FUTURO}

Cuba, en el siglo XXI continúa siendo un país con dificultades económicas, bloqueado, pero con convicciones y propósitos firmes que no se han modificado por muy críticos que sean los tiempos.

El mundo vive hoy etapas de crisis, cuyas causas y consecuencias repercuten sobre la salud. En ella se sinergia los efectos de las crisis energética, alimentaria y financiera junto a las variaciones del clima; todo lo anterior unido a la debilidad que tienen los sistemas de salud de los países más pobres, se convierte en un resultado nefasto para la salud de las poblaciones.

Por lo que, las perspectivas de la colaboración en el futuro y con todas las experiencias acumuladas de casi medio siglo de desarrollo, hacen que uno de sus pilares fundamentales se base en la formación de recursos humanos o sea la creación de capacidades nacionales para que los países receptores de la ayuda, puedan contar con su personal de la salud, proporcionen una mejor cobertura médica y den continuidad a todas las acciones que se realizan en beneficio de la salud de sus poblaciones.

Mientras esto sucede, ya sea en Cuba o en las naciones donde están las Brigadas Médicas, la labor asistencial se mantiene fundamentalmente en la modalidad de PIS, por ser este el que está más acorde a los principios y concepciones del Sistema de Salud cubano, se basa en las acciones de atención primaria y a través de ellas se desarrollan los programas de control específicos que contribuyen a mejorar la situación de salud de las comunidades, son ejemplos los Programas de Reducción de la Mortalidad Materna e Infantil, Lucha contra el VIH/sida, aplicación de la Medicina Natural y Tradicional, desarrollo de investigaciones médicas conjuntas y celebraciones de Foros Científicos de gran valor y aporte, tanto para Cuba como para el país donde se desarrolla la ayuda. 
Se trata de estimular la colaboración Sur-Sur, en la cual Cuba tiene un compromiso político con los países y en la que el aporte consiste en brindar los recursos humanos necesarios, otro país financia la ayuda y el beneficio final es para el receptor, que por supuesto, no cuenta con ninguno de los recursos anteriores.

La transferencia de tecnologías es otro tipo importante de colaboración que se desarrolla por las instituciones del Polo Científico y beneficia a terceros países. Una importante acción en este sentido es la producción de vacuna Antimeningocócica AC mediante un intercambio entre el Instituto Finlay y el de Tecnología e Inmunología de Bio-Manguinhos de Río de Janeiro, Brasil, como respuesta a solicitud de la OMS, para cubrir el déficit de esta vacuna que tenía su producción disminuida por las trasnacionales farmacéuticas. Esta es una importante colaboración Sur-Sur, para ayudar a los 21 países que comprenden el cinturón de la Meningitis en África. ${ }^{23}$

Se estimulan los proyectos de cooperación entre países (TCC), modalidad de cooperación a través de organismos internacionales y el año 2008 se realizó un proyecto que involucró e interrelacionó el trabajo de Cuba-OPS/OMS-Angola, en la estrategia de erradicación de la poliomielitis, con buenos resultados. ${ }^{24}$

Continuar impulsando el Programa Especial de Colaboración con Venezuela y a los procesos de integración que se producen en La Región, con la activa participación de Cuba en el ALBA, como ejemplo de una integración justa y solidaria, encaminada a lograr un intercambio equitativo y sin lucro entre sus países miembros. Continuar impulsando la Operación Milagro, por la gran nobleza y sensibilidad de la propuesta y por los buenos resultados que muestra, además de que proporciona satisfacción para el pueblo cubano y en los países receptores de la ayuda.

El siglo XXI, será otro período de importantes avances en el campo de la colaboración médica como principio básico del Sistema de Salud cubano que encierra uno de los conceptos éticos del pueblo cubano: el internacionalismo.

\section{CONSIDERACIONES FINALES}

Cuba, con los importantes logros alcanzados en la salud ha honrado el centenario de la creación del Ministerio de Salud Pública.

La proyección de la Cooperación Médica como principio básico e indisoluble desde la concepción del Sistema Único de Salud es un aspecto que contribuyó sobremanera a su desarrollo.

Es importante impulsar la Cooperación Médica, encaminada a desarrollar y fortalecer las capacidades nacionales siempre que sus propósitos y principios realmente respondan a las necesidades de cada país, lograr que cada vez sea más sostenible y que exista una continuidad de las acciones de los profesionales cubanos de la salud en los países donde se desarrolla. Su perfeccionamiento trae como principales resultados, la adaptación de metodologías o técnicas ya desarrolladas y probadas en sus lugares de origen, la sistematización de experiencias, transferencia de tecnología y publicaciones de resultados científicos, lo que permite fortalecer los vínculos entre países y alcanzar un desarrollo concreto de sus capacidades institucionales. 
El desarrollo de líneas y estrategias de cooperación conjunta con la OPS/OMS amplía el diapasón y aprovechamiento de oportunidades tanto para Cuba como para la propia Organización, con un objetivo común: el beneficio y mejoría de los indicadores de salud y desarrollo social de sus países miembros. Además, los resultados de esta ayuda se potencian y constituyen una herramienta eficaz para avanzar dentro de los procesos de integración regional en el continente.

Esta labor de ayuda solidaria y desinteresada con otros pueblos del mundo contribuye al perfeccionamiento del Sistema Nacional de Salud, permite fortalecerlo, se adquiere mayor experiencia, conciencia, entrega y compromiso de los profesionales de la salud con ellos mismos y con el pueblo, que les ha depositado se confianza y por supuesto en la Revolución, con la demostración una vez más de que "Patria es Humanidad", como dijo José Martí.

La Colaboración Médica constituye un elemento cardinal en el reconocimiento y respeto internacional que ha ganado Cuba en el mundo en los últimos 50 años, como esencia del proceso revolucionario cubano y de las ideas de Fidel.

\section{REFERENCIAS BIBLIOGRÁFICAS}

1. Delgado García G. Centenario del Ministerio de Salud Pública de Cuba (19092009). Coloquio por el centenario del Ministerio de Salud Pública de CubaLa Habana: Instituto Superior de Ciencias Médicas de la Habana;2009.

2. Delgado García G. La salud pública en Cuba en el periodo de la revolución cubana. Cuad Hist Salud Púb. Conferencia Diez. 1996(81).

3. Delgado García G. Temas y personalidades de la historia de la medicina cubana. Cuad Hist Salud Púb. 1987(72).

4. Delgado García G. Presencia cubana en los orígenes de los Organismos Internacionales de la salud Pública. Cuad Hist Salud Púb [sitio en Internet]. 1995 [citado 16 Jul 2004];80. Disponible en: http://bvs.sld.cu/revistas/his/vol 1 95/his07195.htm

5. Delgado García G. Conferencias de Historia de la Salud Pública en Cuba. Cuad Hist Salud Púb. 1996; (81).

6. Rosell Puig W. Recuerdos de aquel primer viaje [sitio en Internet]. [citado 21 Jul 2005]. Disponible en: http://bvs.sld.cu/revistas/his/cua 88/cua0288.htm

7. de la Torre E, Márquez M, Rojas Ochoa F, Gutiérrez Muñiz JA, López Pardo C. Salud para todos Si es posible. La Habana: Sociedad Cubana de Salud Pública, Sección de Medicina Social; 2005.

8. Castro Ruz F. Discurso pronunciado por el Presidente de la República de Cuba Fidel Castro Ruz, en el acto en la Inauguración del Instituto de Ciencias Básicas y Preclínicas "Victoria de Girón" el 17 de Octubre de 1962 [sitio en Internet]. [citado 2009]. Disponible en:

http://www.cuba.cu/gobierno/discursos/1962/esp/f171062e.html 
9. Delgado García G. La solidaridad internacional de la medicina cubana. Antecedentes, su desarrollo y trascendencia en la etapa revolucionaria. Cuad Hist Salud Púb. 1987;72: 137-49.

10. Jiménez Y. Cuba coopera. Washington,D.C.: OPS;2008.

11. Marimón Torres N. La colaboración Medica Cubana en el siglo XXI: una propuesta para la sostenibilidad en Guinea Bissau [tesis]. La Habana: Escuela Nacional de Salud Pública; 2006.

12. Castro Ruz F. Discurso pronunciado en el encuentro con las fuerzas médicas prometidas para apoyar al pueblo de Estados Unidos en regiones afectadas por huracán Katrina. Palacio de Convenciones. La Habana el 4 de septiembre 2005 [sitio en Internet]. [citado 2009]. Disponible en: http://www.cuba.cu/gobierno/discursos/2005/esp/f040905e.html

13. Gorry C. Cuban Health Cooperation Turns 45. Medicc Review. 2008;10(3).

14. Cuba Coopera. Sitio de Cooperación del Gobierno cubano [sitio en Internet:]. [citado 19 Abr 2010]. Disponible en:

http://www.cubacoop.com/CubaCoop/Inicio.html

15. Castro Ruz F. Discurso pronunciado por el Presidente de la República de Cuba, en la Escuela Latinoamericana de Ciencias Médicas el 3 de diciembre del 2002 [sitio en Internet]. [citado 2009]. Disponible en:

http://www.cuba.cu/gobierno/discursos/2002/esp/f031202e.html

16. Declaración final de la reunión de ministros de salud de Ibero América. Ciudad de La Habana 18 y 19 Octubre 2000 [sitio en Internet]. [citado 19 Abr 2010].

Disponible en: http://www.cumbre-iberoamericana.org/NR/.../III

ReuniondeMinistrosdeSalud.doc

17. Gail A. Training Physicians for Global Health. Medicc Review. 2005; VII(8):1-4.

18. Castro Ruz F. Discurso pronunciado por Fidel Castro Ruz, presidente de la República de Cuba, en ocasión del aniversario 47 de su entrada en Pinar del Río, en el acto por la culminación del montaje de los grupos electrógenos en esa provincia. Pinar del Río el 17 de enero de 2006 [sitio en Internet]. [citado 2009]. Disponible en: http://www.cuba.cu/gobierno/discursos/2002/esp/f031202e.html

19. . Los cristianos sin biblias. Reflexión 3 de marzo de 2008 [sitio en Internet]. [citado 30 Mar 2008]. Disponible en: http://ww.juventudrebelde.cu/cuba/2008-03-03/los-cristianos-sin-biblias/

20. . El destacamento regresa invicto. Reflexión 30 de marzo de 2008 [sitio en Internet]. [citado 30 Mar 2008]. Disponible en: http://ww.juventudrebelde.cu/cuba/2008-03-30/el-destacamento-regresa-invicto/

21. El objetivo irrenunciable. Reflexión 25 de septiembre de 2008 [sitio en Internet]. [citado 25 Sep 2008]. Disponible en: http://ww.juventudrebelde.cu/cuba/2008-09-25/el-objetivo-irrenunciable/

22. Milanés E. Fidel: Principal inspirador de la colaboración médica cubana [sitio en Internet]. [citado 10 Feb 2009]. Disponible en: 
http://new.paho.org/cub/index2.php?option =com docman\&task=doc view\&gid $=20$ 8\&Itemid $=226$

23. Riera L. Ejemplo de colaboración Sur-Sur. Cuba y Brasil suministran vacuna a países africanos, en emergencia por Meningitis [sitio en Internet]. [citado 7 Jun 2008]. Disponible en: http://www.emba.cubaminrex.cu/Default.aspx?tabid $=17723$

24. Montalvo G. Informe Final de la misión de apoyo al Programa de Erradicación de la Poliomielitis en Angola. La Habana: Representación de la OPS/OMS;2008.

Recibido: 7 de mayo de2009.

Aprobado: 1 de febrero de 2010.

Nestor Marimón Torres. Relaciones Internacionales del Ministerio de Salud Pública. Calle 23 No. 177. EL Vedado 10400. La Habana, Cuba.

E-mail: dirdri@informed.sld.cu, evemart@infomed.sld.cu 